\title{
Foreword: Special Issue on IPEC 2014
}

\author{
Marek Cygan ${ }^{1}$ • Pinar Heggernes ${ }^{2}$
}

Received: 23 March 2016 / Accepted: 15 April 2016 / Published online: 22 April 2016 (C) The Author(s) 2016. This article is published with open access at Springerlink.com

We are pleased to present this special issue of Algorithmica, which contains the extended journal versions of selected papers previously presented at the 9th International Symposium on Parameterized and Exact Computation, IPEC 2014, September 10-12, Wrocław, Poland. The symposium is an established annual meeting of the multivariate and exact algorithms communities. This issue consists of nine papers, reviewed thoroughly according to the usual, high standard of the journal.

In The Parameterized Complexity of Geometric Graph Isomorphism, V. Arvind and Gaurav Rattan present improved FPT algorithm for Geometric Graph Isomorphism problem, where one is to decide whether there is a distance preserving bijection between two sets of points in $k$-dimensional euclidian space.

Igor Razgon in On the read-once property of branching programs and CNFs of bounded treewidth proves a space lower bound for non-deterministic read-once branching programs on functions expressible as CNFs with treewidth at most $k$ of their primal graphs.

In Finding Shortest Paths between Graph Colourings, Matthew Johnson, Dieter Kratsch, Stefan Kratsch, Viresh Patel, and Daniël Paulusma give a complete picture of the parameterized complexity of the $k$-colouring reconfiguration problem, where the goal is to modify one proper colouring into another one, by changing the colour of one vertex at a time.

Given a system of linear equations $A x=b$ over the binary field one can ask whether there is a solution of weight at most $t$, exactly $t$ or at least $t$. In Solving Linear

\footnotetext{
$\triangle$ Marek Cygan

cygan@mimuw.edu.pl

1 Institute of Informatics, University of Warsaw, Banacha 2, 02-097 Warsaw, Poland

2 Department of Informatics, University of Bergen, P.O. Box 7803, 5020 Bergen, Norway
} 
Equations Parameterized by Hamming Weight V. Arvind, Johannes Köbler, Sebastian Kuhnert and Jacobo Torán investigate the parameterized complexity of this problem.

Given a pattern $p$ and a text $t$, the goal of the Generalized Function Matching (GFM) problem is to find a mapping from the letters of $p$ to non-empty substrings of $t$, such that applying the mapping to $p$ results in $t$. In A Parameterized Study of Maximum Generalized Pattern Matching Problems Sebastian Ordyniak and Alexandru Popa study the parameterized complexity of the optimization version of GFM under a wide range of parametrizations.

In The Relative Exponential Time Complexity of Approximate Counting Satisfying Assignments Patrick Traxler shows how to reduce the problem of approximate counting satisfying assigments of a CNF formula to the decision version of the problem, while preserving the number of variables of the formula.

Jannis Bulian and Anuj Dawar consider the Graph Isomorphism problem in Graph Isomorphism Parameterized by Elimination Distance to Bounded Degree. The authors prove, that the problem is FPT when parameterized by the number of vertices one needs to remove in order to obtain a graph of bounded maximum degree.

In Polynomial Kernels and User Reductions for the Workflow Satisfiability Problem Gregory Gutin, Stefan Kratsch and Magnus Wahlström investigate the Workshop Satisfiability Problem from the kernelization perspective for the case of regular constraints.

In 2012 Andrew Drucker proved the so called AND-conjecture. Holger Dell in AND-compression of NP-complete problems: Streamlined proof and minor observations presents a streamlined proof of Drucker's theorem.

The editors express their appreciation to the authors and reviewers for contributing to this special issue.

Open Access This article is distributed under the terms of the Creative Commons Attribution 4.0 International License (http://creativecommons.org/licenses/by/4.0/), which permits unrestricted use, distribution, and reproduction in any medium, provided you give appropriate credit to the original author(s) and the source, provide a link to the Creative Commons license, and indicate if changes were made. 